\title{
Ligand-induced IFNGR1 down-regulation calibrates myeloid cell IFNy responsiveness
}

\author{
William J Crisler, Emily M Eshleman, Laurel L Lenz (D)
}

The type II IFN (IFN $\gamma$ ) enhances antimicrobial activity yet also drives expression of genes that amplify inflammatory responses. Hence, excessive IFN $\gamma$ stimulation can be pathogenic. Here, we describe a previously unappreciated mechanism whereby IFN $\gamma$ itself dampens myeloid cell activation. Staining of monocytes from Listeria monocytogenes-infected mice provided evidence of type I IFN-independent reductions in IFNGR1. IFN $\gamma$ was subsequently found to reduce surface IFNGR1 on cultured murine myeloid cells and human CD14 ${ }^{+}$peripheral blood mononuclear cells. IFN $\gamma$-driven reductions in IFNGR1 were not explained by ligand-induced receptor internalization. Rather, IFN $\gamma$ reduced macrophage Ifngr1 transcription by altering chromatin structure at putative Ifngr1 enhancer sites. This is a distinct mechanism from that used by type I IFNs. Ligand-induced reductions in IFNGR1 altered myeloid cell sensitivity to IFN $\gamma$, blunting activation of STAT1 and 3. Our data, thus, reveal a mechanism by which IFNGR1 abundance and myeloid cell sensitivity to IFN $y$ can be modulated in the absence of type I IFNs. Multiple mechanisms, thus, exist to calibrate macrophage IFNGR1 abundance, likely permitting the fine tuning of macrophage activation and inflammation.

DOI 10.26508/Isa.201900447 | Received 4 June 2019 | Revised 23 September 2019 | Accepted 24 September 2019 | Published online 4 October 2019

\section{Introduction}

When microbes penetrate epithelial barriers, host pattern recognition receptors detect microbial or damage-associated host products (PAMPS or DAMPS). Pattern recognition receptor ligation signals the production of cytokines and other factors important for eliciting, shaping, and amplifying inflammatory responses (1, 2). In many cases, microbes are cleared by an initial wave of phagocytes and these inflammatory responses resolve. Persistence of inflammatory responses is associated with chronic conditions such as atherosclerosis, Alzheimer's, and cancer. To better understand and treat these diseases, there is need for an improved understanding of endogenous processes that limit and promote the resolution of inflammatory responses.
One family of cytokines critical for mediating inflammatory responses is the IFNs. Type II IFN (IFNY) is a proinflammatory cytokine that boosts the antimicrobial functions of myeloid cells. IFNy ligates a heterodimeric cell surface receptor, the interferon gamma receptor (IFNGR), comprising ligand-binding IFNGR1 and signaltransducing IFNGR2 proteins (3). Ligation of the IFNGR propagates a signaling cascade involving the Janus tyrosine kinases (JAKs) JAK1 and JAK2. The activated JAKs phosphorylate tyrosine residues in the IFNGR cytoplasmic domain to stimulate recruitment of signal transducer and activator of transcription (STAT) proteins, including STATs 1 and 3. Phosphorylation of STAT1 on Tyrosine 701 (pSTAT1Y ${ }^{701}$ ) induces the formation of canonical PSTAT1 homodimers, which translocate to the nucleus where they bind DNA to promote expression of IFNY-activated genes (GAGs) (4). Many GAGs encode proteins that boost inflammatory responses or increase myeloid cell antimicrobial activities. IFNy stimulation of myeloid cells, thus, plays a critical role in mediating host resistance to infections by numerous intracellular bacteria and parasites $(5,6,7,8)$. Accordingly, defects in the IFNy response increase susceptibility to diverse pathogens, including L. monocytogenes ( $\mathrm{Lm}$ ) and Mycobacterium tuberculosis (Mtb) $(9,10,11,12)$

The type I IFNs comprise IFN $\beta$ and at least 13 other IFN subtypes-all of which ligate the interferon alpha receptor (IFNAR) to elicit cellular responses (13). Abundant production of these cytokines occurs and has been shown to substantially increase host susceptibility during systemic infections by $\mathrm{Lm}$ as well as mucosal infections by $M t b$ and several other bacterial pathogens $(5,14,15,16$, $17,18,19,20)$. These detrimental effects of type I IFNs correlate with their ability to impair myeloid cell responsiveness to IFNy $(5,18,19$, 20, 21). In murine myeloid cells, reduced IFNY responsiveness correlates with rapid silencing of de novo transcription of the Ifngr 1 gene and a subsequent decrease in surface expression of IFNGR1 (22). Reductions in surface IFNGR1 have also been observed on CD14 ${ }^{+}$monocytes from human patients with untreated Mtb (23). Reductions in myeloid cell surface IFNGR1 are associated with silencing of Ifngr 1 transcription due to recruitment of a repressive early growth response factor 3 (EGR3) transcriptional complex to the proximal murine Ifngr promoter (24). Our laboratory recently developed a mouse model in which this repression is circumvented because of transgenic expression of a functional flag-tagged

Department of Immunology \& Microbiology, University of Colorado School of Medicine, Aurora, CO, USA 
IFNGR1 (fGR1) expressed from a macrophage-specific promoter (18). Macrophages from fGR1 transgenic mice maintain IFNGR1 expression despite IFNAR ligation. The increased activation of these fGR1 macrophages by IFNy correlates with increased resistance of fGR1 mice to systemic Lm infection (18). Together, these findings suggest that type I IFN-driven susceptibility to bacterial infections is at least partly due to reductions in myeloid cell IFNy responsiveness.

In the present studies, we noted type I IFN-independent reductions in myeloid cell surface IFNGR1 staining in the context of systemic Lm infection. Further mechanistic investigations demonstrated that this reduced IFNGR1 was driven by IFNY itself and associated with silencing of Ifngr1 transcription. The mechanism for Ifngr 1 silencing was found to be distinct from that used by type I IFNs and involved altered chromatin at putative Ifngr1 enhancer sites. The IFNy-driven reductions in IFNGR were also associated with a transient dampening of myeloid cell responsiveness to IFNY. These data demonstrate that there are multiple mechanisms by which IFNGR1 availability is regulated in myeloid cells, which is counter to the accepted dogma that IFNGR is constitutively expressed. Furthermore, the finding that IFNY acts to impair accessibility of its own receptor suggests this mechanism may be important for increasing the threshold of cytokine required for myeloid cell activation; possibly helping to restrict macrophage activation and inflammatory responses when microbial translocation or other stimuli elicit limited or transient IFNy production.

\section{Results}

\section{L. monocytogenes infection triggers type I IFN-independent reductions in myeloid cell surface IFNGR1}

Our prior studies revealed that type I IFNs drive reductions in myeloid cell surface IFNGR1, correlating with increased susceptibility to i.v. infection with $\operatorname{Lm}(18,22)$. We, therefore, investigated the ability of an antibody that blocks ligation of the type I IFN receptor ( $\alpha$ IFNAR1) to prevent reductions in myeloid cell surface IFNGR1 during Lm infection of C57BL/6 (WT) mice. Groups of mice were treated with $\alpha$ IFNAR1 or, as a control, an antibody that neutralizes IFNY $(\alpha \mid F N \gamma)$. A separate set of mice received both antibodies before infection with $10^{4}$ live Lm. Splenocytes harvested at 0, 24, or 72 hours postinfection (hpi) were stained for surface IFNGR1. Surface IFNGR1 geometric mean fluorescence intensity (GMFI) was reduced by nearly $50 \%$ on gated splenic monocytes (CD11b+Ly6C+Ly6G-) at 72 hpi from the infected control (PBS) mice versus naive controls (Fig 1A). Similar results were obtained in IFN $\gamma$-depleted ( $\alpha \mathrm{IFN} \gamma$ ) mice, whereas no reduction in monocyte IFNGR staining was seen at 72 hpi in mice pretreated with $\alpha$ IFNAR1 or $\alpha$ IFNAR1 plus $\alpha$ IFNy (Fig 1A). These data are consistent with prior work using B6.Ifnar1 ${ }^{-/-}$mice and indicate that blockade of type I IFNs (but not IFNY) suffices to prevent myeloid cell IFNGR down-regulation seen at $72 \mathrm{hpi} \mathrm{(22).}$

Strikingly different results were obtained when we evaluated cell surface IFNGR1 staining on splenic monocytes at 24 hpi (Fig 1B). Here, the reductions in IFNGR1 staining were no longer blocked in the mice treated only with $\alpha$ IFNAR1. Neither were the reductions in IFNGR1 staining blocked in mice treated with $\alpha$ IFNy alone. However, when both $\alpha$ IFNAR 1 and $\alpha$ IFN $y$ were given to the infected mice, myeloid cell surface IFNGR was comparable with that seen in uninfected mice. A similar pattern was seen when IFNGR1 was evaluated on gated splenic DCS (Fig S1A and B). These data indicate that during i.V. Lm infection, cell surface IFNGR1 abundance on these myeloid cells is regulated by both type I IFN-dependent and type I IFN-independent processes. Furthermore, they implicate IFNy itself as a contributor to the type I IFN-independent IFNGR1 downregulation seen during the first $24 \mathrm{~h}$ of systemic Lm infection.

In the context of the Lm infection, the early ( $24 \mathrm{~h}$ ) type I and II IFN-dependent IFNGR1 down-regulation was observed to be consistent with serum IFNy concentrations, which were elevated at 24 hpi but returned nearly to baseline by $72 \mathrm{hpi}$ (Fig 1C). This early IFNy has been shown to derive from antigen-independent $\mathrm{T}$ lymphocyte and NK cells responses to Lm infection (25). The abundance of Ifng transcripts in spleens of the control and infected mice followed similar kinetics (Fig 1D). Conversely, transcript abundance for Ifnb and Ifna subtypes were increased at $24 \mathrm{~h}$ and remained elevated at $72 \mathrm{hpi}$ (Fig $1 \mathrm{E}$ and F). These increases in type I IFN production parallel the increases in bacterial burdens during the i.v. Lm infection (25). Thus, the data argue that the transient spike in IFNY production at $24 \mathrm{hpi}$ contributes together with type I IFNs to the reductions in myeloid surface IFNGR observed at this time point, with later reductions in IFNGR1 being driven solely by the type I IFNS.

\section{IFNy suffices to stimulate reductions in myeloid cell IFNGR1}

To investigate whether IFNy directly impacts myeloid cell surface IFNGR1 abundance, splenocytes from C57Bl/ 6 mice were cultured $8 \mathrm{~h}$ in vitro with $100 \mathrm{U} / \mathrm{ml}$ recombinant IFN $\beta$, IFN $\gamma$, or a combination of both. Treatments with either cytokine alone or in combination sufficed to significantly reduce surface IFNGR1 abundance on gated splenic monocytes (Fig 2A). To test if this effect was unique to murine cells, we next treated PBMCs or THP-1 monocytes with human cytokines and evaluated cell surface IFNGR1 on gated CD14 cells (Figs 2B and C, and S2A). Surface IFNGR1 was also reduced in the treated human myeloid cells. Thus, despite divergence of the human and mouse IFNY and IFNGR1 proteins, IFNy treatment sufficed to reduce IFNGR1 abundance on myeloid cells from both species.

IFNy treatment also significantly reduced surface IFNGR1 staining on BMDMs and BMDCs from WT or B6.Ifnar1 ${ }^{-1-}$ mice (Figs 2D and $\mathrm{S} 2 \mathrm{~B}$ ), indicating the effects of IFNy did not require type I IFN signaling. Comparison of staining of BMDMs from WT mice and those lacking IFNGR1 (Ifngr|- ${ }^{-/}$) further confirmed specificity of the IFNGR1 staining (Fig S2C). However, IFNy stimulation of WT splenocytes did not reduce IFNGR1 staining on T cells (Fig S2D). Interestingly, IFNy stimulation also failed to significantly reduce surface IFNGR2 staining on BMDMs (Fig S2E), arguing the ligated receptor complex was not simply internalized. We further observed that treatments with either IL-6 or IL-10 were unable to reduce IFNGR1 nor IFNGR2 staining at concentrations that sufficed to induce STAT phosphorylation (Fig S2E and F). These data reveal that IFNy is sufficient to reduce myeloid cell surface availability of IFNGR1 and that this response is not universally induced by other pro- or anti-inflammatory cytokines. 
A

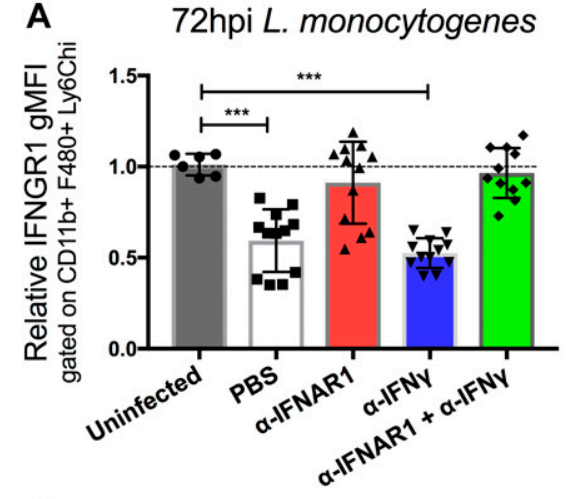

C

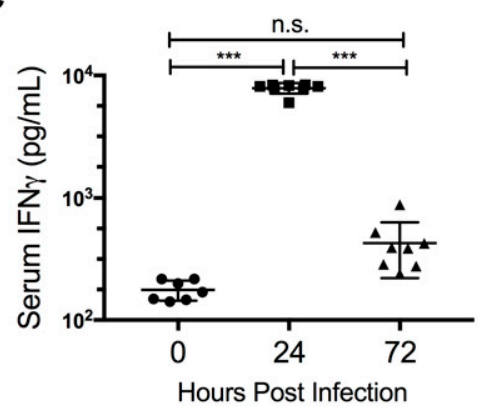

E

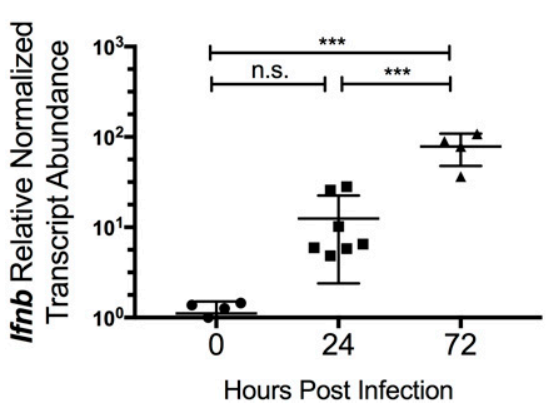

B 24hpi L. monocytogenes

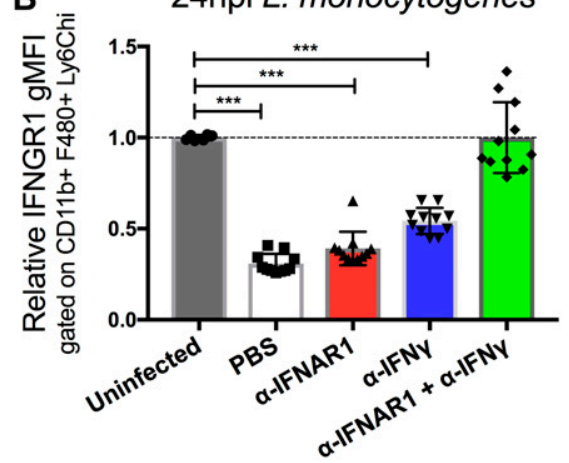

D

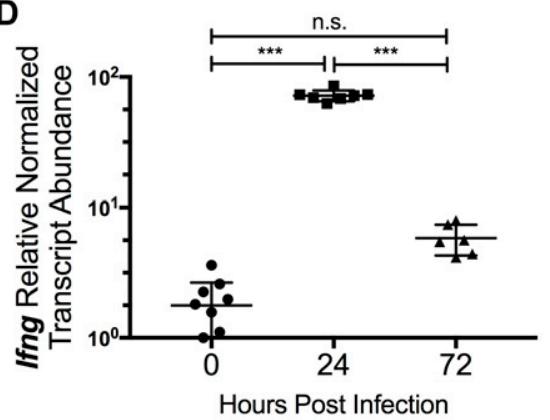

$\mathbf{F}$

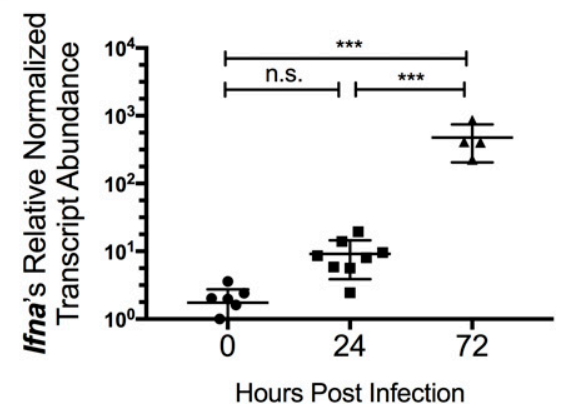

Figure 1. Infection triggers type I IFN-independent reductions in myeloid cell surface IFNGR1 that are dependent on type II IFN (IFN $\gamma$ ).

WT C57BL/ 6 mice were injected i.p. with $0.5 \mathrm{mg}$ of $\alpha$-IFN $\gamma$, $\alpha$-IFNAR1, both antibodies, or PBS vehicle control 24 h before i.v. infection with $10^{4}$ CFU L. monocytogenes. (A, B) At 0, 24, or $72 \mathrm{hpi}$, splenocytes were harvested for FACS analysis. Splenic monocytes were gated as detailed in the Materials and Methods section. Relative changes in $\mathrm{gMFI}$ for IFNGR1 staining is shown versus control staining for three pooled experiments at each time point. (C) Serum IFNy concentrations at the indicated times after infection. Data are pooled from three experiments with a total of 7-9 mice/group. (D, E, F) Relative normalized transcript abundance of Ifng, Ifnb, or Ifna subtypes from lysed whole splenocytes at indicated times after infection. Data are pooled from three experiments with a total of 4-9 mice/group. (A, B, C, D, E, F) For all panels, error bars represent SEM; ${ }^{* * *} \mathrm{P}<$ 0.001 as determined by one-way ANOVA and Dunnett's (A, B) or Tukey's (C, D, E, F) post-hoc tests for comparison between uninfected and other groups or comparison between conditions. n.s., not significant.

\section{Reduced IFNGR1 staining requires JAK signaling and does not correlate with induction of myeloid cell death}

Time course experiments further revealed that reductions in IFNGR1 did not occur immediately following IFNy stimulation, but in WT, BMDMs required at least $2 \mathrm{~h}$ of stimulation and continued to decline for at least $8 \mathrm{~h}$ (Fig S2G). These kinetics suggested that signaling through the IFNGR drives the observed reductions in surface IFNGR1 protein. To test this further, we treated cells with ruxolitinib (Ruxo), an ATP-competitive inhibitor primarily targeting JAK1 and JAK2 (26, 27). Ruxo pretreatment of BMDMs blocked the ability of IFNy to induce down-regulation of IFNGR1 (Fig S2H). These results confirm a requirement for signaling downstream of the IFNGR and further demonstrate that ligand binding to IFNGR is not sufficient to cause the observed reduction in staining of cell surface IFNGR1. Hence, reduced IFNGR1 staining was not due to blockade of antibody binding by the IFNy ligand.

Given the ability of type I and II IFNs to induce apoptosis in susceptible cells $(28,29)$, we considered the possibility that induction of cell death might contribute to reductions in IFNGR1 staining. To evaluate this, BMDMs were treated with a fixable live/ dead stain before surface staining. After excluding doublets and debris, BMDMs were gated on live cells. After $8 \mathrm{~h}$ in culture, $88 \%$ of untreated BMDMs were viable (Fig S2I). Viability of the BMDMs was similarly high in the cultures treated $8 \mathrm{~h}$ with $100 \mathrm{U} / \mathrm{ml}$ IFN $\beta$ or IFNy alone (Fig S2I). Only when IFN $\beta$ and IFN $y$ treatments were combined was there a reduction in cell viability ( $78 \%$ viable). The percentage of live cells was reduced further with increased concentrations of the IFNs but remained $>60 \%$ even with cytokine concentrations of $1,000 \mathrm{U} / \mathrm{ml}$ (Fig S2I). By comparison, only $16 \%$ of heat-killed BMDMs stained within the live gate (Fig S2I). When IFN-treated BMDMs from these cultures were gated on the live-stained population, reductions in IFNGR1 staining were similar regardless of the presence of dead cells in the cultures (Fig S2J). Thus, we conclude that reduced IFNGR1 staining is not an artifact of cell death induction by the IFNs.

\section{IFN $y$ stimulation reduces total cellular abundance of IFNGR1}

Ligation of various cell surface transmembrane receptors is known to trigger receptor-ligand endocytosis (30), and prior studies provided 
A

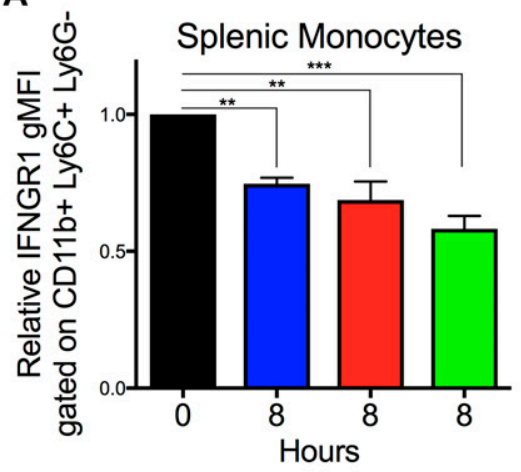

B

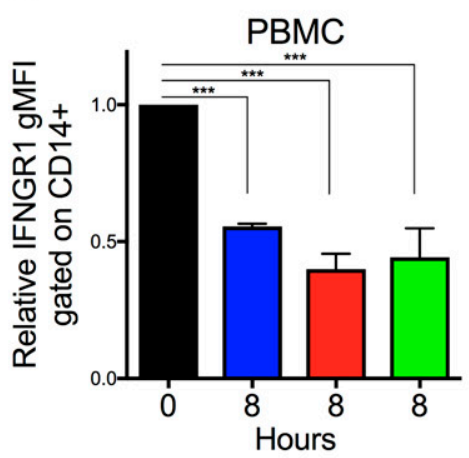

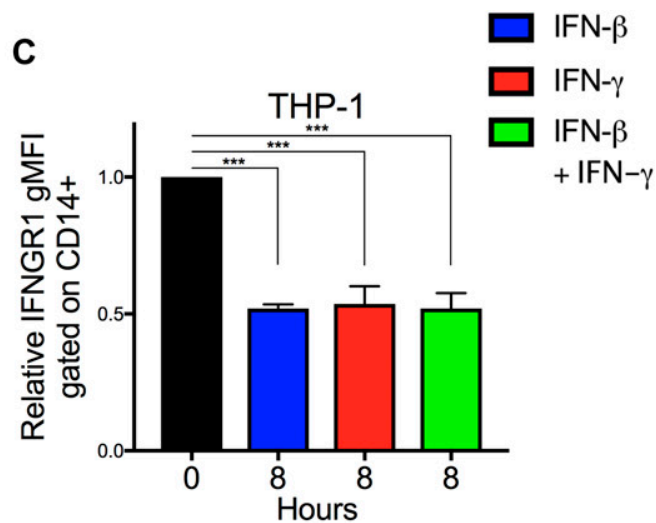

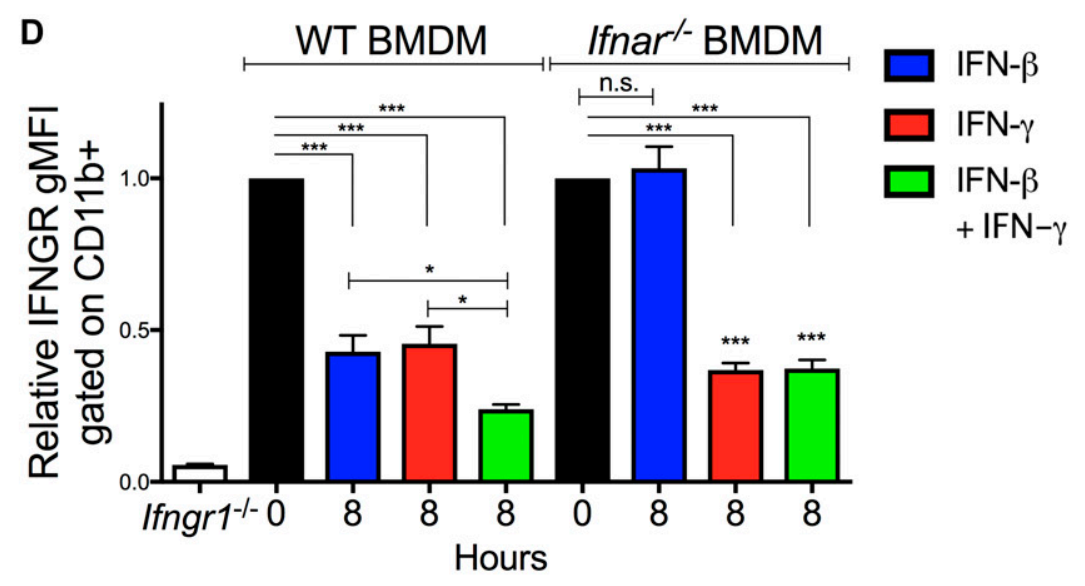

E

WT BMDM

Ifnar'- BMDM

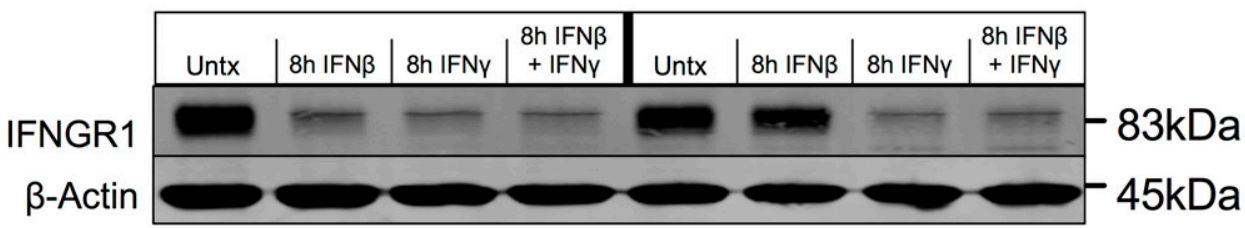

Figure 2. IFN $y$ stimulation reduces surface IFNGR1 abundance on murine and human myeloid cells.

(A, B, C, D) Indicated myeloid cells were treated in culture for $8 \mathrm{~h}$ with $100 \mathrm{U} / \mathrm{ml}$ recombinant IFN $\beta$ (blue), IFNy (red), or IFN $\beta$ and IFNy together (green) then stained and analyzed for cell surface IFNGR1. (A, B, C, D) Shown is normalized surface IFNGR1 gMFI on gated (A) CD11b+, Ly6C+, Ly6G- splenic monocytes from naïve WT mice, (B) $\mathrm{CD}_{14}{ }^{+}$human PBMC, (C) CD14 ${ }^{+}$human THP-1 cells, and (D) CD11 ${ }^{+}$BMDMs from WT or B6.Ifnar1 ${ }^{-1-}$ mice. Each bar graph represents the mean \pm standard deviation (SD) of the pooled values for each condition ( $n=3$ independent experiments). Error bars represent SEM; $n . S .,{ }^{*} P<0.05$, ${ }^{* *} P<0.01$, ${ }^{\star * *} P<0.001$ by one-way ANOVA and Dunnett's or Tukey's post-hoc test for comparison between untreated ( $0 \mathrm{~h}$ ") and other groups or comparison between multiple conditions. (E) WT or Ifnar ${ }^{-/-}$ BMDMs were treated $\pm 8 \mathrm{~h} 100 \mathrm{U} / \mathrm{ml}$ recombinant IFN $\beta$, IFNy, or IFN $\beta$ and IFNy together. Representative immunoblot of lysates probed for IFNGR1 and $\beta$-actin as loading control. n.s., not significant.

evidence for ligand-induced endocytosis of IFNGR1 in epithelial cells (31). Older studies using radio-labeled IFNy also observed that ligation of IFNGR in macrophages transiently reduced the number of cell surface cytokine binding sites (32), consistent with either endocytosis or reduced expression of IFNGR. We, thus, further considered whether IFNy-stimulated reductions in myeloid cell surface IFNGR1 might solely be due to ligand-induced internalization of IFNGR1 protein by evaluating the effects of IFN $\beta$ or IFNy stimulation on total cellular IFNGR1. BMDMs from WT or Ifnart ${ }^{-1-}$ mice were lysed $8 \mathrm{~h}$ after treatment with $100 \mathrm{U} / \mathrm{ml}$ IFN $\beta$, IFNy, or IFN $\beta$ plus IFNy. Total cellular IFNGR1 protein was quantified by immunoblot analysis. Compared with untreated WT cells, total IFNGR1 protein was reduced by $>50 \%$ after $8 \mathrm{~h}$ treatment in each stimulation (Figs 2 E and S3A). Total IFNGR1 protein was also reduced in Ifnar ${ }^{-1-}$ BMDMs, but only in response to the IFNy treatment (Figs 2E and S3A). These data indicate that the loss of surface IFNGR1 in IFNy-stimulated macrophages is not simply due to transient receptor internalization but is instead associated with reduced cellular abundance of this protein. 


\section{IFN $\gamma$ reduces Ifngr1 transcript abundance via a distinct mechanism from type I IFN}

Given that there were reductions in total cellular IFNGR1 after IFNy treatment and type I IFNs are known to silence de novo transcription of the Ifngr1 gene $(22,24)$, we used quantitative real-time PCR (qRT-PCR) to evaluate abundance of Ifngr 1 transcripts at $0-8 \mathrm{~h}$ after IFN $\beta$ or IFN $y$ stimulation. The results indicated that treatment with either IFN type significantly reduced Ifngr1 transcript abundance (Fig 3A). Interestingly, the reduction in Ifngr1 transcript was more rapid after IFN $\beta$ ( $50 \%$ reduction at $\sim 3.8 \mathrm{~h}$ ) versus IFNy $(50 \%$ reduction at $\sim 6 \mathrm{~h}$ ). The delayed effects of IFNy likely reflect the attenuated myeloid cell response to IFNy in the presence of endogenous type I IFNs (5) because IFNy treatment reduced IfngrI transcript abundance much more rapidly ( $50 \%$ reduction at $\sim 2 \mathrm{~h}$ ) in Ifnar1 $^{-1-}$ BMDMs (Fig 3B). IFNy stimulation did not reduce Ifngr2 transcript abundance (Fig S3B), indicating a specific effect on Ifngr1 by IFNy. Treatments with IL-6 or IL-10 failed to reduce Ifngr1 or Ifngr2 transcript abundance (Fig S3B). Thus, similar to type I IFNs, stimulation with IFNY acts to rapidly reduce myeloid cell Ifngr1 mRNA abundance.

To further investigate the mechanisms for reduced Ifngr1 transcript abundance, we performed chromatin immunoprecipitation (ChIP) experiments in the control and treated WT BMDMs. Phosphorylation of RNA pol II at serine five residues within the C-terminal domain heptapeptide repeat is required for transcription initiation (33). We, thus, used ChIP to assess recruitment of pS5-RNA pol II to the Ifngr1 promoter. Compared with control cells where Ifngr1 is actively transcribed, pS5-RNA pol II occupancy at the Ifngr1 transcriptional start site was significantly reduced by IFNy treatment (Fig 3C). This result suggested IFNy acts to block de novo Ifngr1 transcription. Consistent with this, treatment of WT BMDMs with IFNy reduced Ifngr1 transcript abundance as rapidly and effectively as treatment with the RNA pol II inhibitor actinomycin D (Fig S3C). IFN $\beta$ treatment likewise blocks de novo transcription of Ifng $r$, in this case by promoting recruitment of a repressive Egr3/Nab1 complex to a site in the proximal ifngr1 promoter (24). We, thus, evaluated the effects of IFNy treatment on luciferase production by RAW264.7 macrophage reporter cells (IFNGR1pr-luc) stably transfected with a proximal ifngr1 promoter-luciferase construct that includes the Egr binding site (24). Unlike IFN $\beta$, IFNy treatment failed to suppress luciferase reporter activity in these IFNGR1pr-luc cells (Fig 3D). Consistent with this result, ChIP experiments revealed that IFNy treatment of BMDMs did not enrich Egr3 protein at the Ifngr1 promoter in WT BMDMs (Fig 3E). These results indicate that although type I and II IFNs both silence Ifngr1 transcription in myeloid cells, they do so via distinct mechanisms.

To further define how IFNy treatment blocks Ifngr1 transcription, we evaluated occupancy of enhancer regions upstream of the Ifngr1 locus that were identified based on analysis of a published genome-wide H3K4me3 ChIP-seq analysis in human monocytes (34). H3K4me3 accumulation at enhancer sites is associated with active transcription (35). Chromatin was isolated from control, IFN $\beta$, or IFNy-treated THP-1 human monocytes and ChIP performed with an anti-H3K4me3 antibody (Fig 3F). These experiments confirmed association of H3K4me3 at all four tested enhancer regions in control BMDMs and revealed that treatment with IFNy (but not IFN $\beta$ ) selectively and significantly reduced $\mathrm{H} 3 \mathrm{~K} 4 \mathrm{me} 3$ occupancy at two of these regions (one and three in Fig 3F). Neither treatment affected H3K4me3 association at regions 2 or 4 (Fig 3F). These findings together indicate that the rapid silencing of de novo Ifngr1 transcription in IFNy-treated cells is uniquely associated with changes in occupancy or accessibility of enhancer elements upstream of the Ifngr1 gene.

The data above suggested that IFNY suppresses myeloid cell IFNGR1 abundance by blocking de novo transcription of Ifngr1. To further test this, we investigated the ability of IFNy treatment to reduce IFNGR1 staining on splenic monocytes from fGR1 mice, whose macrophages express a transgenic Ifngr 1 gene driven by the $c$-fms promoter (18). IFNy treatment of splenic monocytes from the fGR1 mice did not significantly reduce IFNGR1 staining (Fig 3G). These data suggest that the transgenic Ifngr1 resists silencing by IFNY and, thus, prevents IFNy from reducing surface IFNGR1.

\section{IFN $\gamma$-stimulated myeloid cells become refractory to STAT activation in response to secondary IFN $\gamma$ exposure}

We next considered whether ligand-induced reductions in IFNGR1 sufficed to limit macrophage responsiveness to subsequent IFNY stimulation. Immunoblotting was first used to quantify the duration of PSTAT1Y ${ }^{701}$ induction after a 30-min pulse of IFNy. WT BMDMs were treated for $30 \mathrm{~min}$ with $100 \mathrm{U} / \mathrm{ml}$ IFNy, washed with PBS, and allowed to rest in cytokine-free media before lysis and immunoblotting. This IFNY pulse rapidly induced PSTAT1Y ${ }^{701}$ accumulation in the BMDMs with the abundance of PSTAT1Y ${ }^{701}$ decaying to nearly background levels at $5 \mathrm{~h}$ after stimulation (Fig 4A). We, thus, chose to re-expose BMDMs to a secondary IFNy "hit" (100 U/ml for 5, 10, 30, or $60 \mathrm{~min}) 5 \mathrm{~h}$ after an initial IFNy pulse. Strikingly, the secondary IFNy treatment failed to induce pSTAT1Y ${ }^{701}$ (Figs 4B and S4A). By contrast, when IFN $\beta$ was used in the secondary hit, PSTAT1Y ${ }^{701}$ was potently induced (Figs 4C and S4B). These data indicate there was continued availability of STAT1 in the IFNy-pulsed cells and susceptibility of this STAT1 to tyrosine phosphorylation. Induction of pSTAT3Y ${ }^{705}$ was also abrogated in IFNy-pulsed BMDMs upon secondary "hit" with IFN $y$ but not IFN $\beta$ (Fig S4C). Thus, the IFN $\gamma$-primed cells became refractory to subsequent IFN $\gamma$. This refractoriness of WT BMDMs to IFNy correlated with reduced intensity of cell surface IFNGR1, as measured by flow cytometry (Fig 4D). Because surface staining for IFNGR1 was restored by $12 \mathrm{~h}$ of rest after the initial IFNy pulse (Fig S2G), we further evaluated the ability of IFNy to induce PSTAT1Y ${ }^{701}$ at this later time point. The responsiveness to IFNy was restored (Fig 4D). In contrast to WT peritoneal macrophages (Fig S4D), IFNy-pulsed peritoneal macrophages isolated from fGR1 mice still induced PSTAT1Y ${ }^{701}$ upon the secondary hit with IFNy (Fig 4E), consistent with their ability to retain surface IFNGR1 (Fig 3G). These data together suggested that an initial encounter with IFNy renders WT macrophages unresponsive to IFNy or raises their threshold for responsiveness to this cytokine. To distinguish between these possibilities, we evaluated whether increasing the IFNy concentration used in the secondary stimulation facilitated the induction of PSTAT1Y ${ }^{701}$ or PSTAT3Y ${ }^{705}$. These experiments showed that after an initial IFNy pulse, the amount of IFNy required for a comparable pSTAT1Y $^{701}$ or PSTAT3Y ${ }^{705}$ response $5 \mathrm{~h}$ later was increased by threefold (Figs $4 \mathrm{~F}$ and S4E). We interpret these data to indicate that 

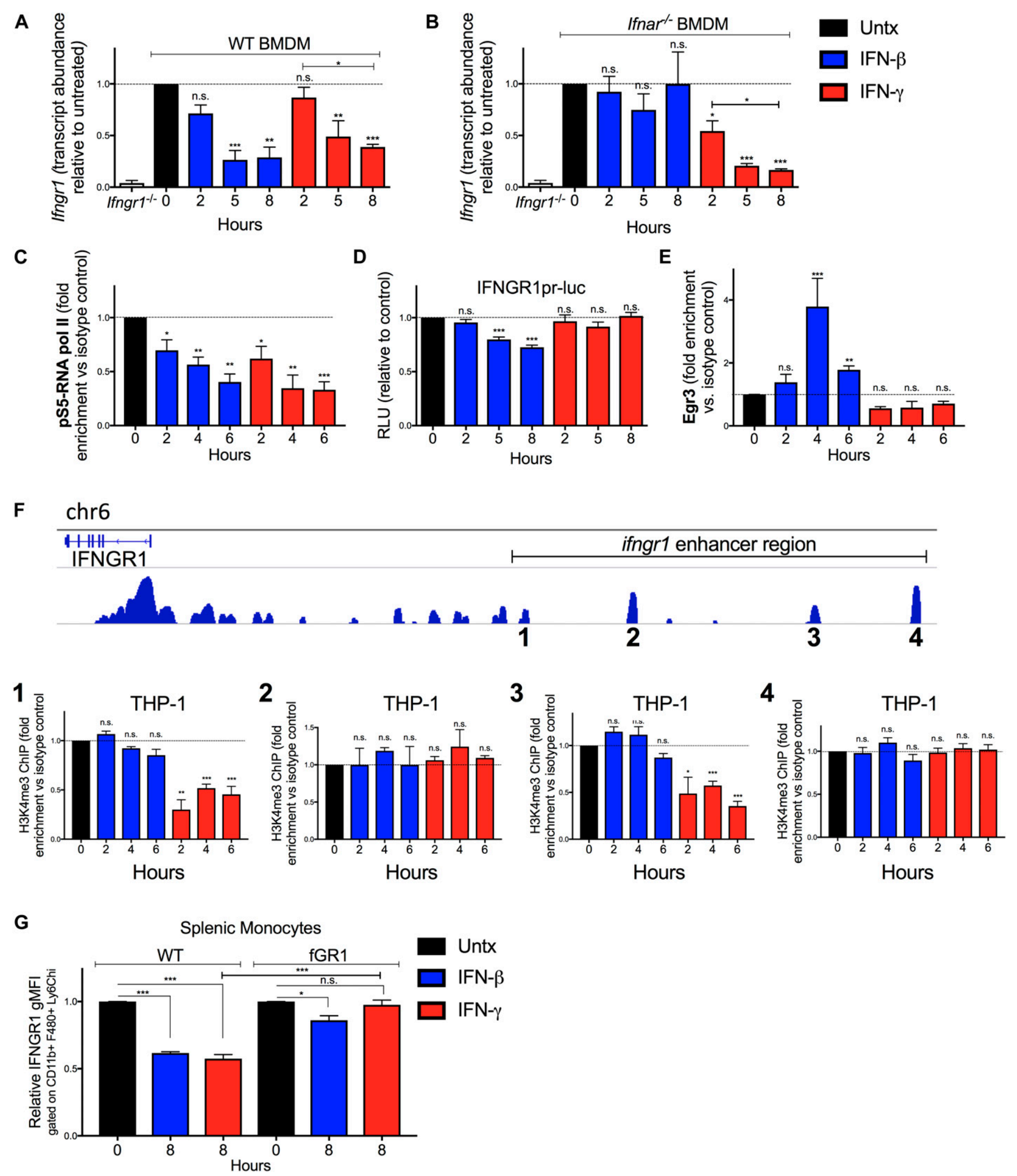

Figure 3. IFN $\gamma$ and IFN $\beta$ reduce Ifngr1 transcript abundance via distinct mechanisms.

WT or Ifnar ${ }^{1 /-}$ BMDMs or RAW 264.7 reporter cells were treated $0-8 \mathrm{~h}$ with $100 \mathrm{U} / \mathrm{ml}$ recombinant IFN $\beta$ (blue) or IFNy (red). (A, B) Total RNA was isolated and analyzed by qRT-PCR. The relative transcript abundance of Ifngr1 was calculated using the $2^{-\Delta \Delta C t}$ model with Gapdh and Hmbs mRNA as housekeeping genes. (C) ChIP was performed for pS5-RNA pol II in WT BMDMs. Murine primers that amplify 100 base pairs within exon 1 of Ifngr1 were used to quantify immunoprecipitated chromatin by qRT-PCR. 
IFNy-induced reductions in myeloid cell surface IFNGR1 act to selectively and transiently dampen myeloid cell sensitivity to subsequent IFNY.

\section{Discussion}

More than 35 years ago, IFNy was demonstrated to play a vital role in the induction of microbicidal and tumoricidal macrophage activation $(36,37)$. However, the complex processes governing macrophage activation remain incompletely defined. Excessive IFNy responses contribute to destruction of host tissue and are associated with a variety of inflammatory diseases (38, 39, 40, 41). IFNy signaling in myeloid cells also broadly impacts hematopoiesis (42). Mechanisms that dampen IFNy responses are, thus, essential for homeostasis. Previously described mechanisms that attenuate cellular responses to IFNy include posttranslational ubiquitination of signal mediators and GAGs, epigenetic changes, and induction of suppressive long noncoding RNAs $(43,44,45,46)$. Our results here have identified an additional, previously unappreciated mechanism whereby IFNy itself acts to tune myeloid cell activation through suppressing Ifngr1 transcript abundance and surface IFNGR1. This rapid suppression of Ifngr 1 is associated with altered occupancy of Ifngr1 enhancer elements and drives a transient reduction in IFNGR1 surface availability that renders macrophages refractory to a second exposure with similar or lesser amounts of IFNy. These findings add a new layer of complexity to our understanding of the IFNy system and the regulation of IFNy-driven myeloid cell activation.

There are similarities between the IFN $y$-driven suppression of IFNGR1 described here and results from our previous work showing that type I IFNs suppress myeloid cell responsiveness to IFNy through suppression of IFNGR1 $(22,24)$. However, there are also important mechanistic differences. Specifically, the silencing of Ifngr1 transcription by IFNy does not involve the recruitment of repressive Egr3 complexes to the proximal Ifngr1 promoter. The existence of two distinct mechanisms for silencing Ifngr1 by type I versus type II IFNs enables finer control of receptor expression during inflammatory responses and seemingly facilitates an increased level of IFNGR1 suppression when the two mechanisms are combined. Although not explored here, the combined suppression of IFNGR1 by type I and II IFNs may render macrophages refractory to higher concentrations of IFNy for a longer time period. This could explain why both mechanisms of suppression are needed and have been conserved throughout the divergent evolution of the IFN systems in mice and humans. These areas will be important for study in future work.

Our previous work showed that silencing of myeloid cell Ifngr1 expression correlates with the detrimental impact of type I IFNs on the host's ability to resist systemic Lm infection (5, 22, 24). Type I IFNs have also been shown to increase the susceptibility to diverse additional systemic and mucosal bacterial infections (5). A transgenic strategy to circumvent this IFNGR1 down-regulation in myeloid cells was, thus, pursued and shown to boost monocyte activation and improve host resistance to systemic Lm (18). Although we initially attributed this protective effect to the reversal of IFNGR1 suppression by type I IFNs, the finding here that fGR1 macrophages similarly circumvent IFNy-driven IFNGR1 downregulation suggests that the resistance of fGR1 mice may reflect the reversal of this process as well. The ability of IFNy to dampen myeloid cell IFNGR1 may also be important to ensure dampening of macrophage responsiveness in the context of infections where pathogen burden is insufficiently high to drive a strong type I IFN response (47). Clearly, further studies will be needed to distinguish between the relative contributions of type I versus type II IFN-driven regulation of IFNGR1 in mediating susceptibility to various infections.

Given the importance of IFNy in mediating inflammation during an innate immune response and the potency of this molecule, conserved mechanisms evolved to control expression of IFNy itself $(48,49,50)$. The work here further shows that modulation of IFNGR1 by IFNY itself is an evolutionarily conserved mechanism to calibrate macrophage responsiveness to IFNy. Our data further indicated that the threshold for STAT protein activation is calibrated during macrophage activation by IFNy through modulation of surface IFNGR1 abundance. This argues that initial exposure of macrophages to IFNy establishes a checkpoint to ensure full macrophage activation only occurs in the presence of persistent or increasing IFNy. This likely helps explain why a prolonged exposure to IFNy is required for full macrophage activation (32) and may have evolved to ensure transient IFNy production in response to "non-dangerous" PAMPS or DAMPs or a limited translocation of microbes at epithelial barriers does not elicit an overly severe inflammatory response. Transient IFNy does trigger STAT activation and drive alterations in myeloid cell gene expression, however. This initial IFNy stimulus corresponds to a "priming" event that can shape the subsequent myeloid cell response. Interestingly, previous studies showed that priming with IFNy dampened macrophage responses to LPS exposure and exerted anti-inflammatory effects (51). Monocytes primed in vivo by limited IFNy production were also shown to play a regulatory role in the context of a parasite infection (52). These

(D) Luciferase activity from lysates of RAW 264.7 reporter cells with luciferase driven by the proximal Ifngr1 promoter. Luciferase activity values were normalized to those of the respective untreated cells. Values were derived from three separate experiments using two independently transfected Ifngr 1 promoter-luciferase cell lines. Statistical significance indicates comparison of relative light units between unstimulated ("0 h"; dashed line) and stimulated groups by one-way ANOVA and Dunnett's post hoc test. (C, E) WT BMDMs were treated as in (C). ChIP assays were performed for Egr3. Murine primers that amplify an Egr site in the proximal Ifngr1 promoter were used to quantify immunoprecipitated chromatin by qPCR. (C, F) Human THP-1 cells were treated as in (C). ChIP assays were performed for H3K4me3. Human primers that amplify putative enhancer regions upstream of the Ifngr1 promoter were used to quantify H3K4me3-associated chromatin by qPCR. (C, E, F) Graphs depict fold enrichment over isotype values normalized to those of the respective unstimulated cells (relative fold enrichment over isotype = fold enrichment from treated sample/average fold enrichment from untreated sample). (G) Naïve WT and fGR1 splenic monocytes were treated for $8 \mathrm{~h} \pm 100 \mathrm{U} / \mathrm{ml}$ recombinant IFN $\beta$ (blue) or IFNy (red). Graph depicts relative gMFI IFNGR1 on gated splenic monocytes. For each panel, bar graph represents the mean \pm SD of pooled values per condition ( $n=3$ independent experiments); $n . S .{ }^{*} P<$ $0.05,{ }^{* \star} P<0.01,{ }^{* *} P<0.001$ by one-way ANOVA and Dunnett's or Tukey's post-hoc test for comparison between untreated and other groups or comparison between conditions. n.s., not significant. 
A WT BMDM

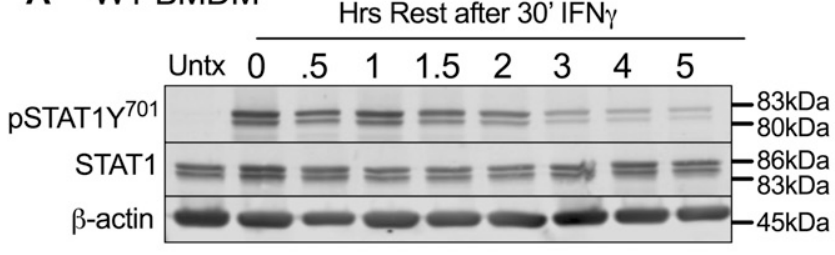

B WT BMDM

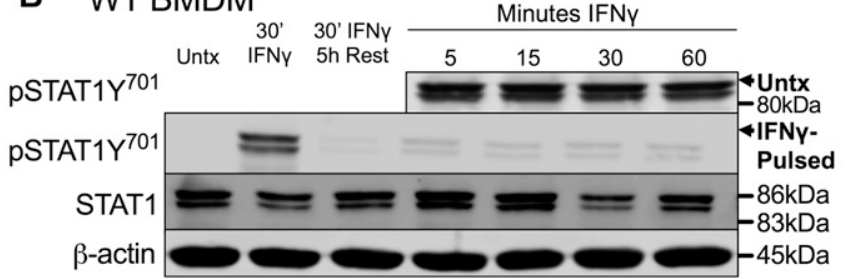

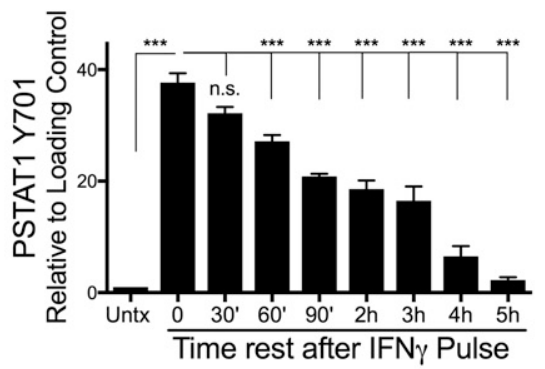

C WT BMDM

30' IFNy 30' IFNy

30' IFNy 5h Rest 5h Rest Untx 30' IFNy 5h Rest 30' IFNy 30' IFN $\beta$

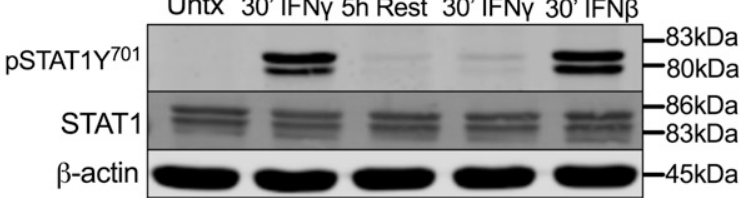

D WT BMDM

$30^{\prime}$ IFN $\gamma$

30' IFNy 30 ' IFN $\gamma$ 5h Rest 30' IFN $\gamma$ 12h Rest

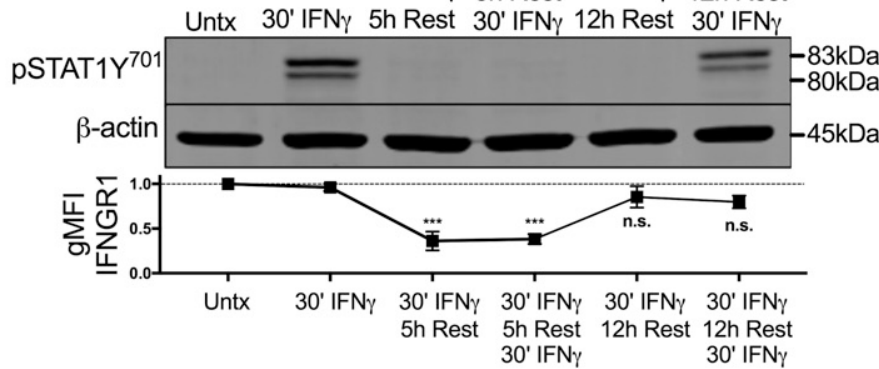

E fGR1 Peritoneal Macrophages

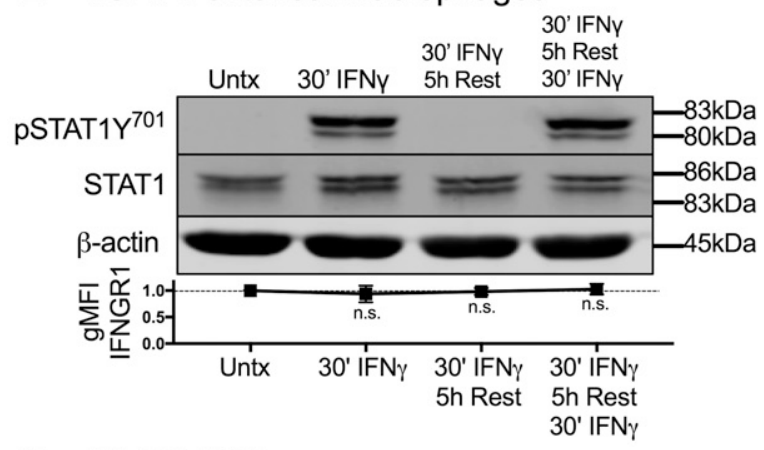

F WT BMDM

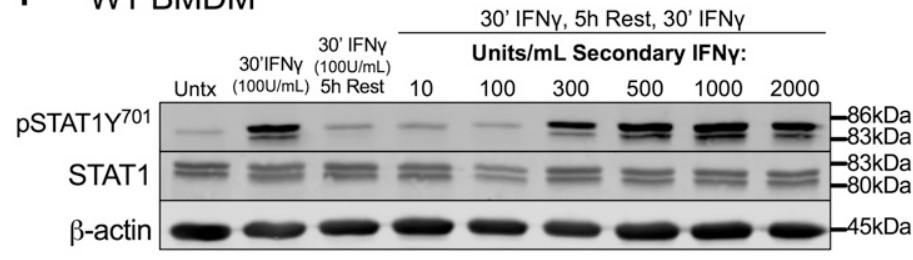

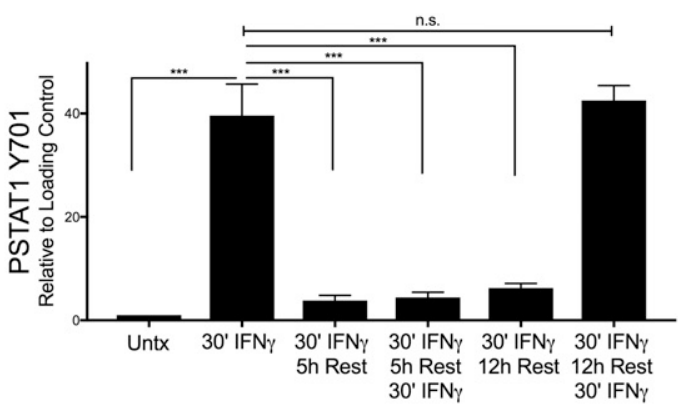
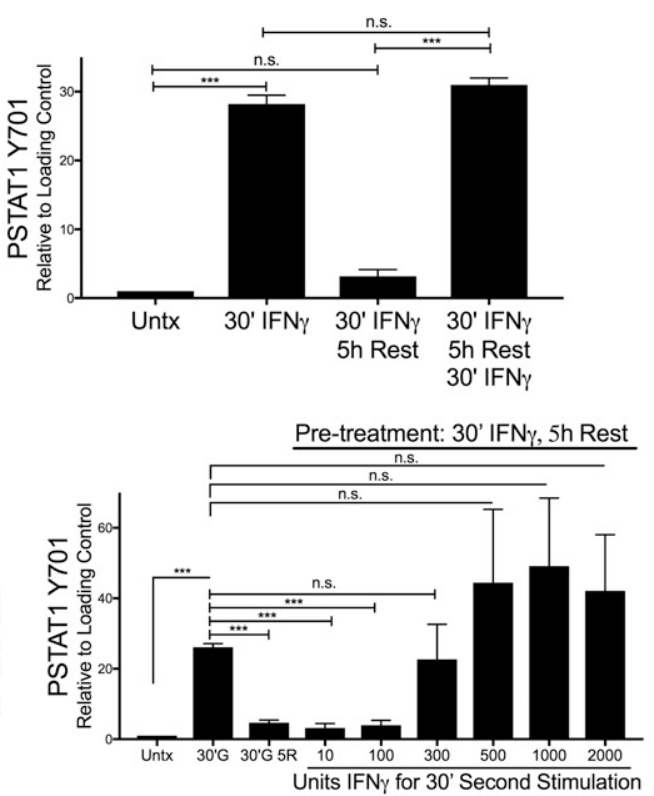

Figure 4. IFN $\gamma$-stimulated myeloid cells become refractory to subsequent IFN $\gamma$-induced PSTAT1Y ${ }^{701}$

WT BMDMs or fGR1 peritoneal macrophages were stimulated and lysed. Representative immunoblots depict lysates probed for pSTAT1Y ${ }^{701}$, Total STAT1, and $\beta$-actin. (A)

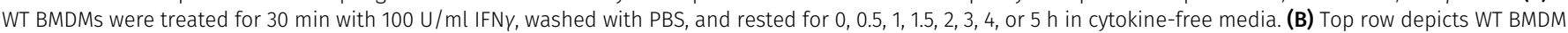
lysates probed at $5,15,30$, or 60 min post stimulation with IFNy with no pretreatment ("Untx," right side). Second row depicts pSTAT1Y ${ }^{701}$ from the following treatments: lane 1(L1), untreated ("Untx"); L2, 30 min (30') IFNy; L3-L7, pretreated with 30' IFNy "pulse," PBS wash, $5 \mathrm{~h}$ rest in cytokine-free medium followed by secondary stimulation "hit" of $100 \mathrm{U} / \mathrm{ml}$ IFN $y$ for 0, 5, 15, 30, or 60 min. (C) WT BMDM lysates probed from the following treatments: lane 1 (L1), untreated (“Untx"); L2, 30 min (30') IFNy; L3, pretreated with 
findings are consistent with the notion that gene expression triggered by a limited IFNY stimulation may primarily have an inflammation dampening effect.

In summary, our results have expanded mechanistic understanding of how myeloid cell responses to IFNy are regulated and provide novel insights regarding the impact and likely importance of IFNGR1 down-regulation in the control of myeloid cell activation, tolerance, and susceptibility to infectious and inflammatory diseases. Nevertheless, there are several limitations of our studies. First, although we observed IFNGR1 down-regulation in a widely used in vivo infection model and showed that IFNY could drive this response in cultured mouse and human cells, it remains to be seen precisely how this regulatory mechanism impacts myeloid cell activation and host resistance in this or other infection models. Second, whereas we showed that IFNy stimulation alters occupancy of enhancer regions upstream of the Ifngrl gene and this correlates with gene silencing, reduced IFNGR1, and impaired responses to subsequent IFNy, proving of direct cause-effect relationships will require additional experimentation. Future work will also be needed to identify specific targets and strategies to therapeutically manipulate or exploit myeloid cell Ifngr1 expression towards enhancing or dampening of myeloid cell activation in the context of infectious, cancerous, or inflammatory disease settings.

\section{Materials and Methods}

\section{Mice}

Adult male and female mice were used at 8-12 wk of age. WT C57BL/ 6 and B6.Ifngr- ${ }^{-/-}\left(\mathrm{Ifngr}^{-/-}\right)$mice were from Jackson laboratory and B6.Ifnar1 ${ }^{-/-}\left(\right.$Ifnar1 $\left.^{-/-}\right)$mice were described previously (22). fGR1 mice were previously described (18). All mice were maintained in a specific pathogen-free colony in the University of Colorado Anschutz Medical Campus Office of Laboratory Animal Research.

\section{Bacterial infections}

L. monocytogenes (Lm; strain 10403s) was thawed from frozen stocks and grown to log phase $(\mathrm{OD} 600=0.1)$ in tryptic soy broth (MP Biomedicals), supplemented with $50 \mu \mathrm{g} / \mathrm{ml}$ of streptomycin. Lm was diluted in PBS and $10^{4}$ CFUs were injected to mice i.v. in the lateral tail vein. For cytokine depletion experiments, monoclonal antibodies were diluted in PBS to a concentration of $2.5 \mathrm{mg} / \mathrm{ml}$. Each mouse received $0.5 \mathrm{mg}$ of antibody in $200 \mu \mathrm{l}$ by intraperitoneal (I.P.) injection $24 \mathrm{~h}$ after infection. IFNy was depleted using $\alpha$-IFNy (XMG1.2; BioXcell). Type I IFNs signaling was blocked using $\alpha$-IFNAR1 (MAR-1; BioXcell). Spleens were harvested into RP10 media (complete Roswell Park Memorial Institute [RPMI] media [Gibco] supplemented with $10 \%$ FBS, $1 \%$ sodium pyruvate, $1 \%$ L-glutamine, and $1 \%$ penicillin/streptomycin) at 0,24 , or $72 \mathrm{hpi}$ then transferred to a digestion solution of $1 \mathrm{mg} / \mathrm{ml}$ of collagenase type IV in HBSS plus cations (Gibco). $1 \mathrm{ml}$ of digestion solution was injected into each spleen. After a 25 -min incubation at $37^{\circ} \mathrm{C}, 0.5 \mathrm{mM}$ EDTA was added to suppress collagenase activity and spleens were physically disrupted and washed through a 70- $\mu \mathrm{M}$ cell strainer with RPMI + P/S. The cell suspensions were next treated with RBC lysis buffer $(0.15 \mathrm{M}$ $\mathrm{NH}_{4} \mathrm{Cl}, 10 \mathrm{mM} \mathrm{KHCO}$, and $0.1 \mathrm{mM} \mathrm{Na} \mathrm{mLDTA}_{2} \mathrm{pH}$ 7.4) for $3 \mathrm{~min}$, quenched with $10 \mathrm{ml} \mathrm{RP10}$, and centrifuged at $500 \mathrm{~g}$ for $5 \mathrm{~min}$. Splenocytes were then analyzed by FACS.

\section{Flow cytometry}

Mouse cells were incubated in anti-CD16/32 (2.4G2 hybridoma supernatant) to block Fc receptors then stained by incubation in FACS buffer (1\% BSA, 0.01\% NaN3, PBS) containing fluorophorelabeled antibodies to mouse proteins that included: anti-CD11b (M1/70; eBioscience), anti-CD11C (N418; BioLegend), anti-Ly6C (Hk1.4; eBioscience), anti-Ly6G (1A8; BioLegend), anti-CD90.2 (53-2.1; eBioscience), anti-IgM (II/4I; eBioscience), and anti-F480 (CL-A3-1; Bio-Rad). Biotinylated anti-IFNGR1/CD119 (2E2; BD Bioscience) and biotinylated anti-IFNGR2 (REA381; Miltenyi Biotec) were stained with secondary streptavidin-APC (eBioscience). For live/dead staining, the cells were stained with LIVE/DEAD Fixable Aqua Dead Cell Stain Kit (\#L34965; Thermo Fisher Scientific) before Fc block and surface staining. Monocytes were gated as live singlet cells staining positive for CD11b, F4/80, and Ly6C and negative for Ly6G. For human cells, anti-CD14 (61D3; BD Biosciences), CD3 (OKT3; BD Biosciences), CD19 (HIB19; BD Biosciences), and CD119/IFNGR1 (GIR-208; Thermo Fisher Scientific) were used. After surface staining, the washed cells were fixed in 2-4\% paraformaldehyde then analyzed using a FACSCalibur (BD Biosciences) or LSR Fortessa (BD Biosciences). Flow data were processed using Flowjo software (TreeStar).

\section{Serum cytokine analysis}

To quantify IFNy production, serum was obtained by allowing blood from cardiac puncture to clot for $15 \mathrm{~min}$ at room temperature. After centrifugation in polypropylene tubes (Sarstedt), serum was collected and transferred to a new microtube and stored at

30' IFNy "pulse," PBS wash, $5 \mathrm{~h}$ rest in cytokine-free media; L4, as in L3 followed by secondary “hit" of $30^{\prime}$ ' $100 \mathrm{U} / \mathrm{mL}$ IFNy; L5, as in L3 followed by secondary "hit" of 30' 100 U/mL IFN B. (D) WT BMDM lysates probed from the following treatments: lane 1 (L1), untreated (“Untx"); L2, 30 min (30') IFNy; L3, pretreated with 30' IFNy "pulse," PBS wash, $5 \mathrm{~h}$ rest in cytokine-free medium; L4, as in L3 followed by secondary "hit" of 30' $100 \mathrm{U} / \mathrm{ml}$ IFNY; L5, pretreated with 30' IFNy "pulse," PBS wash, $12 \mathrm{~h}$ rest in cytokine-free medium; L6, as in L5 followed by secondary "hit" of 30' $100 \mathrm{U} / \mathrm{mL}$ IFNY. (E) fGR1 peritoneal macrophages probed from the following treatments: lane 1 (L1), untreated (“Untx"); L2, 30 min (30') IFNY; L3, pretreated with 30' IFNy “pulse," PBS wash, 5 h rest in cytokine-free medium; L4, as in L3 followed by secondary "hit" of 30' $100 \mathrm{U} / \mathrm{ml}$ IFNY (F) Top row depicts WT BMDM lysates probed for PSTAT1Y ${ }^{701}$ from the following treatments: lane 1 (L1), untreated (“Untx"); L2, $30 \mathrm{~min}$ (30') $100 \mathrm{U} / \mathrm{mL}$ IFNY; L3, pretreated with $100 \mathrm{U} / \mathrm{ml} 30^{\prime}$ IFNy “pulse," PBS wash, 5 h rest in cytokine-free media; L4-L9, as in L3 followed by 30' secondary "hit" with increasing concentrations of IFNY: 10, 100, 300, $500,1,000$, or 2,000 U/ml. For each panel, error bars represent SEM. Each bar graph depicts density of pSTAT1Y ${ }^{701}$ bands normalized to $\beta$-actin $(n=3$ independent experiments). (D, E) Each line graph depicts relative gMFI IFNGR1; n.S., *** $<0.001$ by one-way ANOVA and Dunnett's or Tukey's post-hoc test for comparison between untreated and other groups or comparison between conditions. n.s., not significant.

Source data are available for this figure. 
$-20^{\circ} \mathrm{C}$ until use. Serum IFNy was measured using a commercial ELISA (BD Biosciences).

\section{Cell culture and cytokine stimulations}

To obtain BMDMs, bone marrow was flushed from $\mathrm{C} 57 \mathrm{BL} / 6$, B6.lfngr1 $1^{-/-}$, or B6.Ifnar ${ }^{-/-}$mice femurs and tibias and cultured for $6 \mathrm{~d}$ in BM macrophage media (DMEM supplemented with 10\% FBS, $1 \%$ sodium pyruvate, $1 \%$ L-glutamine, $1 \%$ penicillin/streptomycin, 2mercaptoethanol, and 10\% L-cell conditioned media). Fresh medium was added at day 3. BMDMs were plated on day 6 for use on day 7 of culture. BMDCs were cultured from C57BL/6, Ifngr1 ${ }^{-/-}$, or Ifnar1 ${ }^{-1-}$ mice as previously described (53). RAW 264.7 cells stably transfected with an ifngr1 promoter-luciferase reporter construct (IFNGR1pr-luc) were previously described (24). IFNGR1pr-luc RAW 264.7 murine macrophage cells were cultured in DM10 media (DMEM supplemented with $10 \%$ FBS, $1 \%$ sodium pyruvate, $1 \%$ L-glutamine, and $1 \%$ penicillin/streptomycin). Luciferase activity was measured using a GloMax Microplate Luminometer (Promega). Normalized activity was determined using the formula: (relative luc activity = luc activity from treated sample/average of luc activity from untreated sample). Human monocytic THP-1 cells were cultured in suspension with RP10 media (RPMI 1640 media supplemented with 10\% FBS, 1\% sodium pyruvate, $1 \%$ L-glutamine, and $1 \%$ penicillin/streptomycin). Human PBMCs were isolated from de-identified blood donors. Blood was collected in heparin-containing vacuum tubes, and then white blood cells were separated from whole blood as previously described (24). Peritoneal cells were isolated from fGR1 mice as described previously (18). In brief, $10 \mathrm{ml}$ ice cold PBS was used to lavage the peritoneal cavity. Peritoneal cells were plated in DM10 media on tissue culture-treated plates for several hours to enrich for adherence by macrophages, followed by vigorous washes with room temperature PBS. For cytokine stimulations (unless otherwise noted), murine cells were treated with $100 \mathrm{U} / \mathrm{ml}$ recombinant mouse IFNy (\#714006; BioLegend) or recombinant mouse IFN $\beta$ (\#12401-1; PBL), $10 \mathrm{ng} / \mathrm{ml}$ recombinant IL-6 (\#406-ML-005; R\&D Systems), or $50 \mathrm{ng} / \mathrm{ml}$ recombinant IL-10 (\#14-8101-62; eBioscience). PBMCs and THP-1 cells were treated with $100 \mathrm{U} / \mathrm{ml}$ recombinant human IFNy (\#11500-1; PBL) or recombinant human IFN $\beta$ (\#11410-1; PBL). For inhibition of JAK, WT BMDMs were treated with $5 \mu \mathrm{g} / \mathrm{ml}$ ruxolitinib (\#S1378; Selleckchem) for $1 \mathrm{~h}$. To inhibit transcription, the cells were treated with $1 \mu \mathrm{g} / \mathrm{ml}$ actinomycin D (\#AC29494-0050; Thermo Fisher Scientific).

\section{Immunoblotting}

At designated time points after cytokine stimulation, total cell lysates from BMDMs or BMDCs were washed three times with room temperature PBS. For experiments including cytokine stimulation followed by rest, BMDMs or BMDCs were treated for 30 min with designated concentration of cytokine, washed once with room temperature PBS, and cultured in cytokine-free media for specified time of rest. The cells were lysed in the culture dish using $0.02 \%$ NP40 supplemented with HALT protease and phosphatase inhibitors (Thermo Fisher Scientific) and 1× SDS-PAGE buffer (0.0625 M Tris-Cl, pH 6.8, 2\% SDS, 10\% glycerol, 5\% 2-ME, and 0.01\% bromophenol blue) was added. Equivalent protein amounts were loaded into $10 \%$ acrylamide gels and transferred onto Polyvinylidene difluoride membranes (Millipore). Blots were probed for IFNGR1 (K17), pSTAT1Y $^{701}$ (58D6; Cell Signaling), or Total STAT1 (91-C; Cell Signaling) with $\beta$-Actin (8H10D10; Cell signaling) as a loading control on each blot. Blots were developed using the secondary antibodies goat $\alpha$-rabbit IR 800 (926-32211; LI-COR) and goat $\alpha$-mouse IR 680 (926-68070; LI-COR) and imaged on an Odyssey CLX (LI-COR). All PSTAT1Y ${ }^{701}$ and IFNGR1 bands were normalized to $\beta$-actin on the same blot using ImageStudio ver 4.0 software (LI-COR). Densitometry graphs are pooled from at least three independent PSTAT1Y ${ }^{701}$ or IFNGR1 blots.

\section{RNA isolation, cDNA synthesis, and qRT-PCR}

Total RNA was isolated from splenocytes, BMDMs, or BMDCs in RLT lysis buffer (QIAGEN) and stored at $-80^{\circ} \mathrm{C}$. The cells were disrupted using a 20-gauge needle and syringe and RNA extracted using the RNeasy Mini Kit following the manufacturer's instructions. cDNA synthesis was conducted using the iScript CDNA Synthesis Kit (BioRad). qRT-PCR was performed using the iTaq Universal SYBR Green Supermix (Bio-Rad). The following exon spanning primer sets were used for murine cells: Ifng fw: 5'-AGCTCTTCCTCATGGCTGTT-3', rev: 5'TाTTGCCAGTTCCTCCAGAT; Ifnb fw: 5'-CATCAACTATAAGCAGCTCCA-3', rev: 5'-TTCAAGTGGAGAGCAGTTGAG-3', Ifna subtypes fw:5'-CTTCCACAGGATCACTGTGTACCT-3', rev: 5'-TTCTGCTCTGACCACCTCCC-3'; Gapdh fw: 5'-ATGGCCTCCAAGGAGTAAG-3', rev: 5'-CCTAGGCCCCTCCTGTTATT-3'; Hmbs fw: 5'-GAGTCTAGATGGCTCAGATAGCATGC-3', rev: 5'-CCTACAGACCAGTTAGCGCACATC-3'; Ifngr1 fw: 5'-AGGTGTATTCGGGTTCCTGG-3', rev: 5'-AATACGAGGACGGAGAGCTG-3'; Ifngr2 fw 5'-GTCCTCGCCAGACTCGTTTT-3', rev: 5'-CCCGCAGGAAGACTGTGAAT-3'. All qRT-PCRs were conducted in 384-well format with a total reaction volume of 12 $\mu$ l on a CFX384 Touch Real-Time PCR Detection System (Bio-Rad). The relative transcript abundance values were calculated using the $2^{-\Delta \Delta C t}$ model with GAPDH and HMBS mRNA as internal controls (54).

\section{ChIP}

The ChIP experiments were performed according to the protocol provided for the Active Motif ChIP Express kit (Active Motif) and described previously (24). Briefly, BMDMs or THP-1 cells were cross-linked with $1 \%$ methanol-free formaldehyde for $7 \mathrm{~min}$ at room temperature. Fixed cells $\left(7 \times 10^{6}\right.$ in $\left.300 \mu \mathrm{l}\right)$ were resuspended in kit lysis buffer plus protease inhibitors and incubated at $30 \mathrm{~min}$ at $4^{\circ} \mathrm{C}$. Cell nuclei were pelleted and resuspended in $300 \mu \mathrm{l}$ of kit shearing buffer plus protease inhibitors. A Covaris S2 sonicator was used to shear the samples using a 27-cycle treatment. $10 \mu \mathrm{l}$ of supernatant was saved for use as total input DNA. All samples were stored at $-80^{\circ} \mathrm{C}$ until use. Immunoprecipitations were performed overnight at $4^{\circ} \mathrm{C}$ with protein $\mathrm{G}$ magnetic beads (\#53033; Active Motif) plus $7 \mu \mathrm{g}$ of sheared chromatin and antibodies specific for Egr3 (ab75461; Abcam), pS5-RNA pol II (ab5131; Abcam), H3K4me3 (\#39915; Active Motif) or control antibodies for mouse IgG (ab46540; Abcam), and human IgG (ab2410; Abcam). After immunoprecipitation, the beads were washed and the immune complexes eluted with kit elution buffer. Reverse cross-linking buffer was added to each eluted supernatant at 1:1. Samples and input DNA were heated for $1 \mathrm{~h}$ at $95^{\circ} \mathrm{C}$. After treatment with $10 \mu \mathrm{g} / \mathrm{ml}$ proteinase $\mathrm{K}$ for $1 \mathrm{~h}$ at $37^{\circ} \mathrm{C}$, the samples were purified using QIAGEN PCR 
purification kit and then used for qPCR. The murine Ifngr1 promoter primer sequences used to analyze ChIPs were previously described (24), and their sequences were as follows: (pS5-RNA pol II ChIP) fw: 5'-GCAATTGTGTCCCTCGCGCAGGAATGGGCC-3'， N: 5'-GCTCGTCAAAGCTCCACTCCCGACC-3', (Egr3 ChIP) fw: 5'-CCTCAGGCTAGTCCACCCCTTCTCC-3', rev: 5'-GGAGGCGTGTCTTGGCGGG-3'. Primers used for query of human enhancer region sequences were as follows: (H3K4me3 ChIP) fw: 5'-TGTCTGTCCTTTGAGCGGGA-3', rev: 5'-CTGTCTCAGCAAGTCGAGGA-3'; fW: 5'-ATTCAAACCACAGGCTCCGA-3', rev: 5'-GACTTTGGCCAAGGCATACCA-3'; fW: 5'-AACTCAAAAGCAAGCGCACA-3', rev: 5- TCACTCTCAAGCGAACCTGC-3'; fW: 5'-TCTGCTTTATGGAGCGGCTT-3', rev: 5'-GTGTGCTCGCAAGTGTAACC-3'. qRT-PCR was performed as described above. Graphed results depict fold enrichment over isotype values normalized to those of the respective untreated cells (relative fold enrichment over isotype = fold enrichment from treated sample/average fold enrichment from the untreated sample).

\section{Supplementary Information}

Supplementary Information is available at https://doi.org/10.26508/lsa. 201900447.

\section{Acknowledgements}

The authors thank the current and past members of our laboratory for helpful discussion and input into these studies. We especially thank Dr. Sarah Clark, Dr. Daniel McDermott, and Nikki Bortell. Research funding was provided by National Institute of Allergy and Infectious Diseases (NIAID). NIAID grants R33Al102264, R21Al140499, and R01Al131662 (to LL Lenz). WJ Crisler and EM Eshleman received support from T32AI052066.

\section{Author Contributions}

WJ Crisler: conceptualization, data curation, formal analysis, investigation, methodology, and writing-original draft, review, and editing

EM Eshleman: conceptualization, data curation, formal analysis, and methodology.

LL Lenz: conceptualization, formal analysis, supervision, funding acquisition, project administration, and writing-original draft, review, and editing.

\section{Conflict of Interest Statement}

The authors declare that they have no conflict of interest.

\section{References}

1. Iwasaki A, Medzhitov R (2010) Regulation of adaptive immunity by the innate immune system. Science 327: 291-295. doi:10.1126/science.1183021

2. Mosser DM, Edwards JP (2008) Exploring the full spectrum of macrophage activation. Nat Rev Immunol 8: 958-969. doi:10.1038/nri2448

3. Pestka S, Krause CD, Walter MR (2004) Interferons, interferon-like cytokines, and their receptors. Immunol Rev 202: 8-32. doi:10.1111/j.01052896.2004.00204.x
4. Borden EC, Sen GC, Uze G, Silverman RH, Ransohoff RM, Foster GR, Stark GR (2007) Interferons at age 50: Past, current and future impact on biomedicine. Nat Rev Drug Discov 6: 975-990. doi:10.1038/nrd2422

5. Crisler WJ, Lenz LL (2018) Crosstalk between type I and II interferons in regulation of myeloid cell responses during bacterial infection. Curr Opin Immunol 54: 35-41. doi:10.1016/j.coi.2018.05.014

6. Eshleman EM, Lenz LL (2014) Type I interferons in bacterial infections: Taming of myeloid cells and possible implications for autoimmunity. Front Immunol 5: 431. doi:10.3389/fimmu.2014.00431

7. Arora S, Hernandez Y, Erb-Downward JR, McDonald RA, Toews GB, Huffnagle GB (2005) Role of IFN-gamma in regulating T2 immunity and the development of alternatively activated macrophages during allergic bronchopulmonary mycosis. J Immunol 174: 6346-6356. doi:10.4049/ jimmunol.174.10.6346

8. Flynn JL, Chan J, Triebold KJ, Dalton DK, Stewart TA, Bloom BR (1993) An essential role for interferon gamma in resistance to Mycobacterium tuberculosis infection. J Exp Med 178: 2249-2254. doi:10.1084/ jem.178.6.2249

9. Jouanguy E, Lamhamedi-Cherradi S, Lammas D, Dorman SE, Fondaneche MC, Dupuis S, Doffinger R, Altare F, Girdlestone J, Emile JF, et al (1999) A human IFNGR1 small deletion hotspot associated with dominant susceptibility to mycobacterial infection. Nat Genet 21: 370-378. doi:10.1038/7701

10. Roesler J, Kofink B, Wendisch J, Heyden S, Paul D, Friedrich W, Casanova JL, Leupold W, Gahr M, Rosen-Wolff A (1999) Listeria monocytogenes and recurrent mycobacterial infections in a child with complete interferongamma-receptor (IFNgammaR1) deficiency: Mutational analysis and evaluation of therapeutic options. Exp Hematol 27: 1368-1374. doi:10.1016/s0301-472x(99)00077-6

11. Lee SH, Carrero JA, Uppaluri R, White JM, Archambault JM, Lai KS, Chan SR, Sheehan KC, Unanue ER, Schreiber RD (2013) Identifying the initiating events of anti-listeria responses using mice with conditional loss of IFNgamma receptor subunit 1 (IFNGR1). I Immunol 191: 4223-4234. doi:10.4049/jimmunol.1300910

12. Lykens JE, Terrell CE, Zoller EE, Divanovic S, Trompette A, Karp CL, Aliberti J, Flick MJ, Jordan MB (2010) Mice with a selective impairment of IFNgamma signaling in macrophage lineage cells demonstrate the critical role of IFN-gamma-activated macrophages for the control of protozoan parasitic infections in vivo. J Immunol 184: 877-885. doi:10.4049/ jimmunol.0902346

13. van Pesch V, Lanaya H, Renauld JC, Michiels T (2004) Characterization of the murine alpha interferon gene family. J Virol 78: 8219-8228. doi:10.1128/jvi.78.15.8219-8228.2004

14. Fehr T, Schoedon G, Odermatt B, Holtschke T, Schneemann M, Bachmann MF, Mak TW, Horak I, Zinkernagel RM (1997) Crucial role of interferon consensus sequence binding protein, but neither of interferon regulatory factor 1 nor of nitric oxide synthesis for protection against murine listeriosis. J Exp Med 185: 921-931. doi:10.1084/jem.185.5.921

15. O'Connell RM, Saha SK, Vaidya SA, Bruhn KW, Miranda GA, Zarnegar B, Perry AK, Nguyen BO, Lane TF, Taniguchi T, et al (2004) Type I interferon production enhances susceptibility to Listeria monocytogenes infection. J Exp Med 200: 437-445. doi:10.1084/jem.20040712

16. Carrero JA, Calderon B, Unanue ER (2006) Lymphocytes are detrimental during the early innate immune response against Listeria monocytogenes. J Exp Med 203: 933-940. doi:10.1084/jem.20060045

17. Auerbuch V, Brockstedt DG, Meyer-Morse N, O'Riordan M, Portnoy DA (2004) Mice lacking the type I interferon receptor are resistant to Listeria monocytogenes. J Exp Med 200: 527-533. doi:10.1084/ jem.20040976

18. Eshleman EM, Delgado C, Kearney SJ, Friedman RS, Lenz LL (2017) Down regulation of macrophage IFNGR1 exacerbates systemic $L$. monocytogenes infection. PLoS Pathog 13: e1006388. doi:10.1371/ journal.ppat.1006388 
19. Teles RM, Graeber TG, Krutzik SR, Montoya D, Schenk M, Lee DJ, Komisopoulou E, Kelly-Scumpia K, Chun R, Iyer SS, et al (2013) Type I interferon suppresses type II interferon-triggered human antimycobacterial responses. Science 339: 1448-1453. doi:10.1126/ science. 1233665

20. Manca C, Tsenova L, Freeman S, Barczak AK, Tovey M, Murray PJ, Barry C, Kaplan G (2005) Hypervirulent M. tuberculosis W/Beijing strains upregulate type I IFNS and increase expression of negative regulators of the Jak-Stat pathway. I Interferon Cytokine Res 25: 694-701. doi:10.1089/ jir.2005.25.694

21. McNab F, Mayer-Barber K, Sher A, Wack A, O'Garra A (2015) Type I interferons in infectious disease. Nat Rev Immunol 15: 87-103. doi:10.1038/nri3787

22. Rayamajhi M, Humann J, Penheiter K, Andreasen K, Lenz LL (2010) Induction of IFN-alphabeta enables Listeria monocytogenes to suppress macrophage activation by IFN-gamma. J Exp Med 207: 327-337. doi:10.1084/jem.20091746

23. Singhal A, Jaiswal A, Arora VK, Prasad HK (2007) Modulation of gamma interferon receptor 1 by Mycobacterium tuberculosis: A potential immune response evasive mechanism. Infect Immun 75: 2500-2510. doi:10.1128/iai.01743-06

24. Kearney SJ, Delgado C, Eshleman EM, Hill KK, O'Connor BP, Lenz LL (2013) Type I IFNs downregulate myeloid cell IFN-gamma receptor by inducing recruitment of an early growth response 3/NGFI-A binding protein 1 complex that silences ifngr1 transcription. J Immunol 191: 3384-3392. doi:10.4049/jimmunol.1203510

25. Clark SE, Filak HC, Guthrie BS, Schmidt RL, Jamieson A, Merkel P, Knight V, Cole CM, Raulet DH, Lenz LL (2016) Bacterial manipulation of NK cell regulatory activity increases susceptibility to Listeria monocytogenes infection. PLoS Pathog 12: e1005708. doi:10.1371/journal.ppat.1005708

26. Quintas-Cardama A, Vaddi K, Liu P, Manshouri T, Li J, Scherle PA, Caulder E, Wen X, Li Y, Waeltz P, et al (2010) Preclinical characterization of the selective JAK1/2 inhibitor INCB018424: Therapeutic implications for the treatment of myeloproliferative neoplasms. Blood 115: 3109-3117. doi:10.1182/blood-2009-04-214957

27. Zhou T, Georgeon S, Moser R, Moore DJ, Caflisch A, Hantschel O (2014) Specificity and mechanism-of-action of the JAK2 tyrosine kinase inhibitors ruxolitinib and SAR302503 (TG101348). Leukemia 28: 404-407. doi:10.1038/leu.2013.205

28. Ossina NK, Cannas A, Powers VC, Fitzpatrick PA, Knight JD, Gilbert JR, Shekhtman EM, Tomei LD, Umansky SR, Kiefer MC (1997) Interferongamma modulates a p53-independent apoptotic pathway and apoptosis-related gene expression. J Biol Chem 272: 16351-16357. doi:10.1074/jbc.272.26.16351

29. Apelbaum A, Yarden G, Warszawski S, Harari D, Schreiber G (2013) Type I interferons induce apoptosis by balancing CFLIP and caspase-8 independent of death ligands. Mol Cell Biol 33: 800-814. doi:10.1128/ mcb.01430-12

30. Aguilar RC, Wendland B (2005) Endocytosis of membrane receptors: Two pathways are better than one. Proc Natl Acad Sci U S A 102: 2679-2680. doi:10.1073/pnas.0500213102

31. Marchetti M, Monier MN, Fradagrada A, Mitchell K, Baychelier F, Eid P, Johannes L, Lamaze C (2006) Stat-mediated signaling induced by type I and type II interferons (IFNs) is differentially controlled through lipid microdomain association and clathrin-dependent endocytosis of IFN receptors. Mol Biol Cell 17: 2896-2909. doi:10.1091/mbc.e06-010076

32. Celada A, Schreiber RD (1987) Internalization and degradation of receptor-bound interferon-gamma by murine macrophages. Demonstration of receptor recycling. J Immunol 139: 147-153.

33. Phatnani HP, Greenleaf AL (2006) Phosphorylation and functions of the RNA polymerase II CTD. Genes Dev 20: 2922-2936. doi:10.1101/ gad. 1477006
34. Weiterer S, Uhle F, Lichtenstern C, Siegler BH, Bhuju S, Jarek M, Bartkuhn M, Weigand MA (2015) Sepsis induces specific changes in histone modification patterns in human monocytes. PLoS One 10: e0121748. doi:10.1371/journal.pone. 0121748

35. Pekowska A, Benoukraf T, Zacarias-Cabeza J, Belhocine M, Koch F, Holota H, Imbert J, Andrau JC, Ferrier P, Spicuglia S (2011) H3K4 tri-methylation provides an epigenetic signature of active enhancers. EMBO I 30: 4198-4210. doi:10.1038/emboj.2011.295

36. Nathan CF, Murray HW, Wiebe ME, Rubin BY (1983) Identification of interferon-gamma as the lymphokine that activates human macrophage oxidative metabolism and antimicrobial activity. J Exp Med 158: 670-689. doi:10.1084/jem.158.3.670

37. Pace JL, Russell SW, Torres BA, Johnson HM, Gray PW (1983) Recombinant mouse gamma interferon induces the priming step in macrophage activation for tumor cell killing. I Immunol 130: 2011-2013.

38. Zhang J (2007) Yin and yang interplay of IFN-gamma in inflammation and autoimmune disease. J Clin Invest 117: 871-873. doi:10.1172/jci31860

39. Mehta NN, Teague HL, Swindell WR, Baumer Y, Ward NL, Xing X, Baugous B, Johnston A, Joshi AA, Silverman J, et al (2017) IFN-gamma and TNF-alpha synergism may provide a link between psoriasis and inflammatory atherogenesis. Sci Rep 7: 13831. doi:10.1038/s41598017-14365-1

40. Han JH, Suh CH, Jung JY, Ahn MH, Han MH, Kwon JE, Yim H, Kim HA (2017) Elevated circulating levels of the interferon-gamma-induced chemokines are associated with disease activity and cutaneous manifestations in adult-onset still's disease. Sci Rep 7: 46652. doi:10.1038/srep46652

41. Sarvetnick N, Shizuru J, Liggitt D, Martin L, McIntyre B, Gregory A, Parslow T, Stewart T (1990) Loss of pancreatic islet tolerance induced by betacell expression of interferon-gamma. Nature 346: 844-847. doi:10.1038/ $346844 a 0$

42. de Bruin AM, Voermans C, Nolte MA (2014) Impact of interferon-gamma on hematopoiesis. Blood 124: 2479-2486. doi:10.1182/blood-2014-04568451

43. Kim TK, Maniatis T (1996) Regulation of interferon-gamma-activated STAT1 by the ubiquitin-proteasome pathway. Science 273: 1717-1719. doi:10.1126/science.273.5282.1717

44. Burr ML, Cano F, Svobodova S, Boyle LH, Boname JM, Lehner PJ (2011) HRD1 and UBE2J1 target misfolded MHC class I heavy chains for endoplasmic reticulum-associated degradation. Proc Natl Acad Sci U S A 108: 2034-2039. doi:10.1073/pnas.1016229108

45. Aune TM, Collins PL, Collier SP, Henderson MA, Chang S (2013) Epigenetic activation and silencing of the gene that encodes IFN-gamma. Front Immunol 4: 112. doi:10.3389/fimmu.2013.00112

46. Gomez JA, Wapinski OL, Yang YW, Bureau JF, Gopinath S, Monack DM, Chang HY, Brahic M, Kirkegaard K (2013) The NeST long ncRNA controls microbial susceptibility and epigenetic activation of the interferon-gamma locus. Cell 152: 743-754. doi:10.1016/ j.cell.2013.01.015

47. Pitts MG, Myers-Morales T, D'Orazio SE (2016) Type I IFN does not promote susceptibility to foodborne Listeria monocytogenes. I Immunol 196: 3109-3116. doi:10.4049/jimmunol.1502192

48. Ben-Asouli Y, Banai Y, Pel-Or Y, Shir A, Kaempfer R (2002) Human interferon-gamma mRNA autoregulates its translation through a pseudoknot that activates the interferon-inducible protein kinase PKR. Cell 108: 221-232. doi:10.1016/s0092-8674(02)00616-5

49. Cavalieri RL, Havell EA, Vilcek J, Pestka S (1977) Induction and decay of human fibroblast interferon mRNA. Proc Natl Acad Sci U S A 74: 4415-4419. doi:10.1073/pnas.74.10.4415

50. Young HA, Ghosh P (1997) Molecular regulation of cytokine gene expression: Interferon-gamma as a model system. Prog Nucleic Acid Res Mol Biol 56: 109-127. doi:10.1016/s0079-6603(08)61004-1 
51. Hoeksema MA, Scicluna BP, Boshuizen MC, van der Velden S, Neele AE, Van den Bossche J, Matlung HL, van den Berg TK, Goossens P, de Winther MP (2015) IFN-gamma priming of macrophages represses a part of the inflammatory program and attenuates neutrophil recruitment. J Immunol 194: 3909-3916. doi:10.4049/jimmunol.1402077

52. Askenase MH, Han SJ, Byrd AL, Morais da Fonseca D, Bouladoux N, Wilhelm C, Konkel JE, Hand TW, Lacerda-Queiroz N, Su XZ, et al (2015) Bone-marrow-resident NK cells prime monocytes for regulatory function during infection. Immunity 42: 1130-1142. doi:10.1016/ j.immuni.2015.05.011

53. Schmidt RL, Filak HC, Lemon JD, Potter TA, Lenz LL (2011) A LysM and SH3domain containing region of the Listeria monocytogenes p60 protein stimulates accessory cells to promote activation of host NK cells. PLOS Pathog 7: e1002368. doi:10.1371/journal.ppat.1002368

54. Stephens AS, Stephens SR, Morrison NA (2011) Internal control genes for quantitative RT-PCR expression analysis in mouse osteoblasts, osteoclasts and macrophages. BMC Res Notes 4: 410. doi:10.1186/17560500-4-410

(c)

License: This article is available under a Creative Commons License (Attribution 4.0 International, as described at https://creativecommons.org/ licenses/by/4.0/). 